\title{
Augmented Shared Spaces: An Application for Exposure Psychotherapy
}

https://doi.org/10.3991/ijoe.v16i04.11876

\author{
Bruno Patrão ( $\left.{ }^{\varpi}\right)$, Paulo Menezes, Nuno Gonçalves \\ University of Coimbra, Coimbra, Portugal \\ bpatrao@isr.uc.pt
}

\begin{abstract}
The latest advances in computational power of small portable devices enables the development of new forms of Augmented Realities, namely Augmented Reality Shared Spaces. In this work, we will explore this technology to improve the collaborative work between people in the same physical space allowing them to share and visualise virtual contents. The idea is to have a $3 \mathrm{D}$ scene shared between several people where everyone can see and interact with and explore its use in psychotherapy context. These live interactions can also be used to improve the quality of collaborative work by presenting in real time ideas and thoughts of any participant directly on top of the 3D model. In the case of a psychotherapy session typically patients describe their feelings and fears to the therapist. This may be centred on virtual representations of their phobic elements, supporting interactions with them, being those actions and results observable by both in their shared space. Our main goal is to apply and evaluate this concept, particularly in exposure-based therapies, where it is important for the therapist to control and manipulate the scene that the patient is exposed to in order to obtain the desired emotional activations.
\end{abstract}

Keywords-Mixed Reality, Virtual Shared Spaces, Collaborative Experiences, Psychotherapy Tools

\section{Introduction}

In this work, we explore future technologies and environments that will improve the interaction between humans and computers as well as interaction between humans mediated by computers. Specifically, we evaluate how Augmented Reality enhanced by physical and spatial 3D user interfaces can be used to develop effective face-toface collaborative computing environments. With this technology, computers can provide the same type of collaborative environment that people have in interpersonal interactions, such as communication by object manipulation and gestures.

Human interaction research focus on integrating typically human characteristics (e.g., cognitive, behavioural, and emotional) into a more intuitive, enjoyable, and useful human-machine approach to everyday contexts. In this sense, Mixed Reality is 
a useful technology to build scenarios or situations, in which one can interact to develop useful skills to apply in real environments.

The full spectrum of Mixed Realities [1], presented in Fig. 1, has covered many areas and gone beyond the research or prototypes, entering the field of commercial and practical solutions. The availability of low-cost immersive devices, the growing computing power of portable devices and cloud services create excellent opportunities for the application of immersive systems in therapeutic uses. Psychology is exactly one of the areas that can have a real benefit with these systems, in particular in exposure-based therapies.

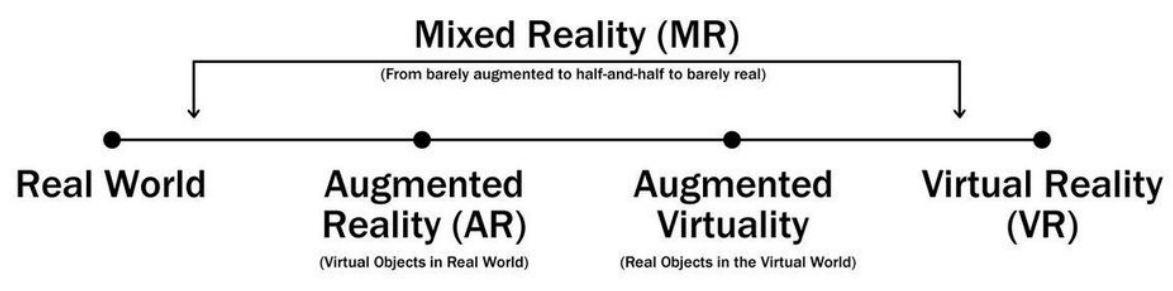

Fig. 1. Mixed Reality Continuum from Milgram \& Kishino (1994).

In Mixed Reality systems, there is a need to bring together various qualities to the scenario in order to increase user's immersion and make the experience vivid and real. One way to assess these qualities of such systems is the user's point of view and subjective experience. Thus, the inclusion of self-report questionnaires to evaluate both the interaction and the realism of the elements in the virtual environment may be an important way of evaluation and validation of these systems.

In the psychology field, the practice of exposure and response prevention is used in the treatment of obsessive-compulsive disorder to deal with compulsive behaviours as a maintenance problem. The most common fears or obsessions include worries about dirt and contamination, which consequently are associated with washing, checking, repeating, ordering / arranging. These compulsive behaviours serve an emotion regulation function and are aimed to reduce anxiety and other difficult emotions (e.g., disgust) [2]. Some psychological disorders involve the experience of high levels of anxiety and distress in face of phobic stimulus (e.g., spiders in the case of arachnophobia; dirty objects and fear of contamination in some obsessive-compulsive disorders). Commonly people tend to avoid and/or escape any phobic stimulus or contexts to achieve a reduction of anxiety and a temporary emotional relief. Nevertheless, this practice tends to reinforce anxiety and avoidance behaviours. In general, psychological treatment involves breaking this vicious cycle, through the exposure of the person to the phobic stimulus in a gradual way [3].

Exposure tasks are planned and set up in a collaborative way between therapist and patient. At the beginning, the therapist acts as a role model in exposure exercises, and then the patient perform the exposure by himself. Some of these fear scenarios for exposure tasks are difficult to design and have in real life, so the aid of complementary technologies may be both helpful and highly attractive for patients. 
Augmented Reality is very useful to present objects coexisting with the physical spaces in a realist and coherent way. Although, most of the experiences are designed for only one person, i.e. the observer, the one with the device that supports the experience. To solve this limitation and continue to take full advantage of Augmented Reality we bring the concept of Shared Spaces to the equation.

Shared Spaces are very relevant to enhance the communication between two or more people. Furthermore, this alliance between Augmented Reality and Shared Spaces allow all the participants to continue interact in a face-to-face way. In communication, particularly when sharing experiences, it is fundamental to have everyone in the same context to guarantee that there are no misleading or bad interpretations about the situation. This is particularly important in psycho-therapeutic environments where the therapist must infer most of the cues from observation. Such system can be a useful tool to aid the therapist in the exposure therapy procedure.

The present work has been motivated by previous research in immersive spaces through Virtual Reality and emotion recognition, where we could find that the interaction with environment was intuitive with a good sense of presence [4]. However, Virtual Reality systems are fully immersive, i.e., they separate the user from the real world. This means that is very difficult to give access to real objects and tools in these immersive Virtual Reality environments. In opposition to Virtual Reality and other traditional computer supported collaborative work, Augmented Reality interfaces are able to overlay computer generated visualizations of objects and audio onto the real world, allowing them to coexist with the physical ones. Furthermore, these 3D objects can have a very realist appearance and be manipulated by multiple the users. More than a simple Augmented Reality application this technology allows everyone to see and share any changes made to both virtual and physical space simultaneously.

\section{Proposed Augmented Reality Shared Space System}

With a special focus on exposure psychotherapy, the proposed system can be very useful for the treatment of several phobias, e.g. arachnophobia (Fig. 2).

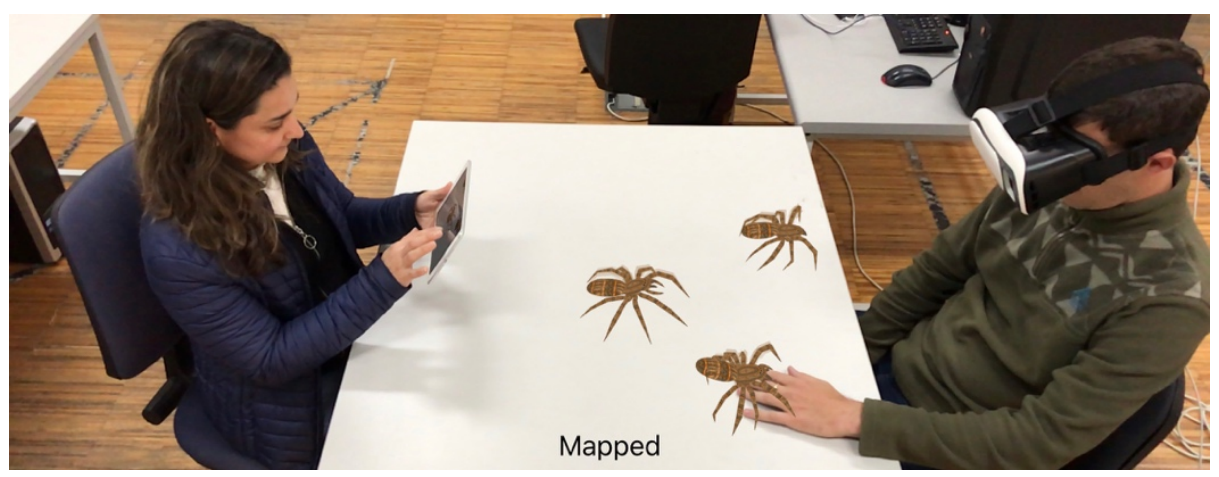

Fig. 2. Shared Space between therapist (on left) and patient (on right). 
The main goal of our system is to create an environment where the therapist can manipulate and share 3D realistic content presented in Augmented Reality with the patients. The therapist can perform the following operations: select, add and remove objects; set scales and positions of objects; define behaviours for each object independently. The manipulations and changes made by the therapist to this virtual environment intend to result in emotional activation and/or behavioural responses from the patients. These responses are the target in the psychological treatment and reducing them without avoidance is the main goal of this kind of intervention.

\subsection{System apparatus}

Our Augmented Reality Shared Space system can be experienced using optical or video see-through devices, such as head-mounted displays, smart glasses or handheld based. For this particular work the devices used were handheld tablets. In addition to these devices the only requirement is a physical surface where the interaction and sharing will occur. Furthermore, the system is prepared to connect and collect data from several physiological devices. Physiological data can also be used as complementary information to data collected from the patient's assessments and therapist observations. Physiological patterns may help by assessing and quantifying stress, anxiety, anger and other emotions that influence physical and mental health. The physiological signals are collected from various modalities such as electroencephalogram (EEG), electrocardiogram (ECG), electromyogram (EMG), galvanic skin response (GSR), blood volume pressure, respiration pattern, and skin temperature [5]. This data is processed and, may not only provide useful cues to the therapist, but also be used to control the system, i.e. if anxiety levels are low during the exposure, the phobic stimuli may be amplified in a controlled way, and vice-versa. For the scope of this work we will not focus on physiological data.

\section{Experiment}

\subsection{Participants}

Nineteen participants (14 males and 5 females) were recruited from different laboratories from our institution at the university campus. Their average age was 28.5 years old $(S D=7.4)$, ranging between 20 and 46 years old. No significant differences were found between males and females regarding age $(p=.343)$. None of the participants had any prior knowledge of the experiment.

\subsection{Material}

The User Experience Questionnaire (UEQ) [6] is a self-report questionnaire that assess the users' opinion about the usability. This scale includes 26 bipolar items organized in six factors, namely Attractiveness (i.e., overall impression of the product), Perspicuity (i.e., easy to get familiar with the product), Efficiency (i.e., the 
degree to which users' solve the tasks without unnecessary effort), Dependability (i.e., the degree to which users' control the interaction), Stimulation (i.e., how exciting and motiving the users' feel when using the product), and Novelty (i.e., evaluation of how innovative and creative the product is). Participants are asked to answer contrasting attributes after the experiment (e.g., attractive versus unattractive) with a 7-points scale. The values per item are transformed to an interval between -3 and +3 . In the original study, it was found adequate to high internal consistency: Dependability $\alpha=$ .65 with the lower reliability value and Attractiveness $\alpha=.89$ with the highest reliability value. In the present study we have found the following Cronbach alphas: Attractiveness $\alpha=.88$; Perspicuity $\alpha=.81$; Stimulation $\alpha=.78$; Novelty $\alpha=.71$; Dependability $\alpha=.64$; Efficiency $\alpha=.42$.

The ITC-Sense of Presence Inventory (ITC-SOPI) [7] is a self-report questionnaire to originally assess the sense of presence in virtual scenarios, defined as the user's subjective sensation of "being there" in media experience. This scale comprises four factors: Sense of Physical Space, Engagement, Ecological Validity, and Negative Effects. Scores of each factor should be individually analysed. Following a media experience, participants answered each item or statement with a 5-point Likert scale $(1=$ strongly disagree to $5=$ strongly agree). Higher scores represent higher subjective experience of each ITC-SOPI's factor. For the present study's purpose, only two factors were chosen to assess participants' experiences after and during the experiment, namely Engagement and Ecological Validity. The Engagement subscale (13 items) refers to individuals' tendency to be psychologically engaged in and involved with the content of the displayed environment (e.g., "I enjoyed myself."). The Ecological Validity subscale (5 items) refers to the tendency to evaluate the virtual environment as believable and real (e.g., "I had a strong sense that the characters and objects were solid."). In the original study, this scale revealed good internal consistency with Cronbach alphas superior to .70 as recommended (Engagement $\alpha=.89$; Ecological Validity $\alpha=.76$ ). In addition, these two factors seem to significantly discriminate between different media scenarios, suggesting that this scale can provide reliable and valid results. In the present study both subscales demonstrated a good internal consistency: Engagement $\alpha=.85$ and Ecological Validity $\alpha=.82$.

\subsection{Experimental procedure}

To evaluate our Augmented Reality Shared Space system, we split the experiment in two parts. The first part of the experimental procedure will test the interaction and usability while the second part will focus on the engagement and ecological validity (coherence and realness of the scene). After each part, all participants were asked to fill an assessment to evaluate that particular part. Handheld devices used by the participant and the instructor to interact with the environment were tablets connected and synchronised to each other sharing information, such as, positions and actions. 


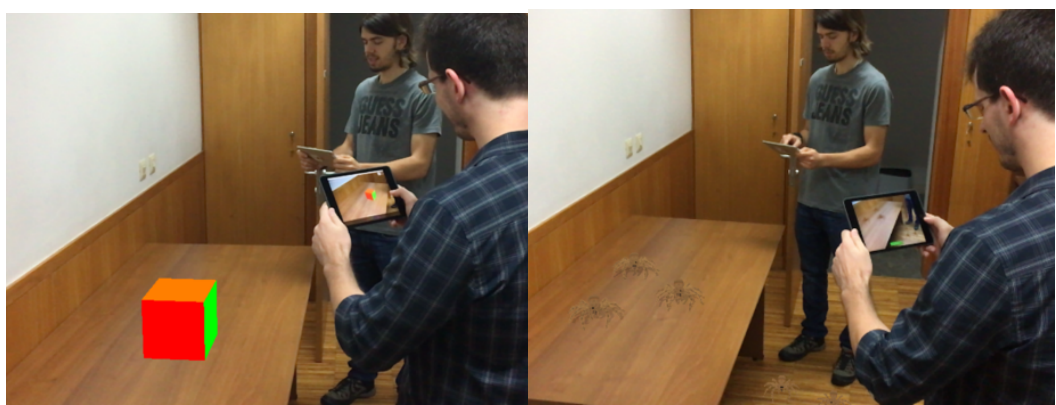

Fig. 3. Part 1 - Usability and Interaction (on left); and Part 2 - Engagement and Ecological Validity (on right).

Part 1 - The first part of the experiment starts with a virtual object placed on top of the table in front of both the participant and the instructor. One can manipulate the object and the other will see the changes. Furthermore, the object can be manipulated by both at the same time. The first task was to rotate and scale the object through pan and pinch gestures, then the instructor does the same and ask if they could perceive the actions performed. The second task involves selecting and colourising different parts of the object in a specific order suggested by the instructor (Fig. 3, left). Finally, the participant and the instructor could freely manipulate the object for a short period of time before filling the assessment to evaluate the interaction and usability. The questionnaire used for this part was the UEQ - User Experience Questionnaire [6].

Part 2 - The second part of the experiment begins with one virtual spider placed on top of the table where both can see and manipulate using their devices. Then the instructor asks the participant to analyse the scene and look closely to the spider from different points of view. Once the participant finish inspecting the environment around the scene the instructor asks to place four more spiders in specific positions marked on the table. While the participant is doing this task, the instructor, secretly places a few more spiders on the floor behind the participant and asks to inspect the environment again after placing the spiders, expecting to surprise the participant. In this experiment the spiders had four different behaviours: they move towards the participant if they are too far away (more than 1 meter); they move away from the participant if they get too close (less than 0,5 meters); they stay in an idle state if the participant is nether close or far from them (more than 0,5 meters and less than 1 meter); and they are always facing towards the participant position (Fig. 3, right). Finally, the participant could freely inspect and place more spider in the environment before filling the assessment to evaluate the engagement and ecological validity. The questionnaire used for this part was the ITC - Sense of Presence Inventory [7].

\section{$4 \quad$ Results}

By splitting the experiment in these two parts we are able to individually evaluate, on the one hand, the interaction module (i.e., object manipulation) and if it is suitable 
to this type of Augmented Reality shared systems in general, on the other hand, the graphical and behavioural module (i.e., scene realness) by measuring the participants' perception of the scene and how engaged they felt.

Regarding the first part of the experiment, results presented in Fig. 4 indicate that participants evaluated the interaction module with high scores on Perspicuity $(M=$ $2.45, S D=0.32)$, Efficiency $(M=2.03, S D=0.39)$, Attractiveness $(M=1.97, S D=$ $0.45)$, Stimulation $(M=1.93, S D=0.58)$, Novelty $(M=1.53, S D=0.76)$, and Dependability $(M=1.30, S D=0.82)$. Overall, participants perceived the augmented shared space interaction as easy to get familiar with, understandable, practical, and enjoyable. The lower mean score of the Dependability scale, in comparison with other scales, may be due to the nature of the shared space experience. Given that both users manipulate simultaneously the same virtual object, the user's control perception may be diminished.

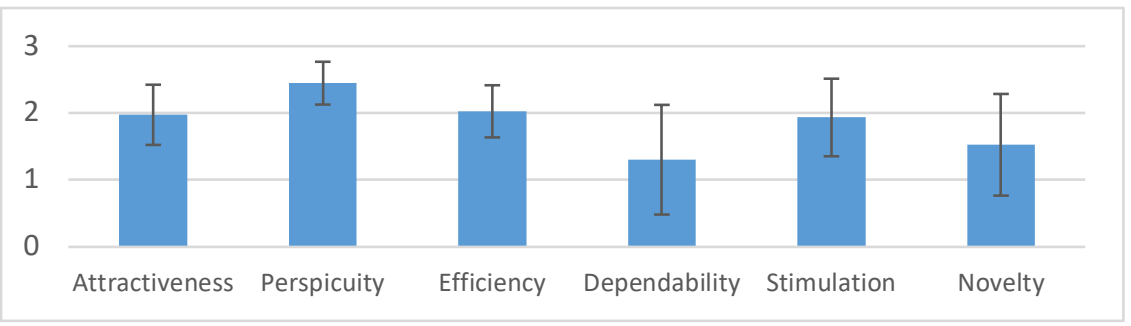

Fig. 4. UEQ results obtained from the first part of the experience.

Results from the second part of the experiment showed a positive evaluation of the graphical and behaviour module. As can be seen in Fig. 5, the Engagement scale had a mean score of $3.96(S D=0.56)$ and Ecological Validity had a mean score of $3.93(S D$ $=0.77)$. Results also indicate a significant and positive correlation between both scales $\left(r_{s}=.46, p=.05\right)$, which mean that participants who evaluate the shared space environment as believable and real tend to feel more engaged and involved in the experience.

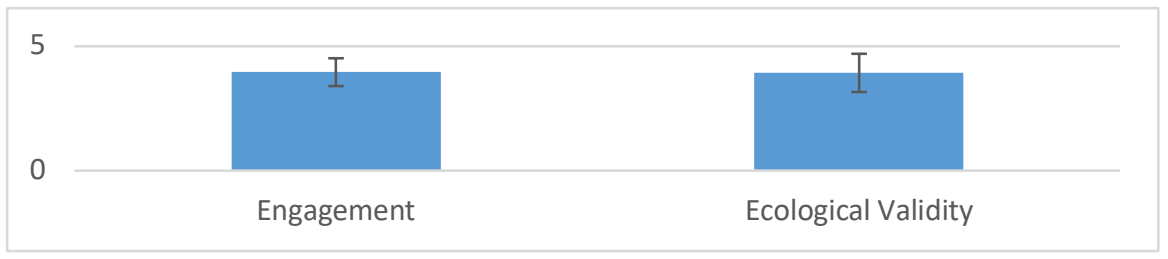

Fig. 5. ITC-SOPI results obtained from the second part of the experiment.

\section{Conclusion}

In this work, we combine real and virtual environments to create coherent and realist $3 \mathrm{D}$ collaborative experiences in psychotherapy context. The system is intended 
to aid the therapist with patient's emotional understanding by creating situations and scenarios that were not possible to conduct in purely real live environments.

Overall, the results of the present study indicated that the participants provided positive indicators about the interaction and usability of the system. Additionally, the more realness and vividness characteristics of the virtual objects perceived by the participants, the more engaged they were.

\section{References}

[1] P. Milgram, and F. Kishino, A taxonomy of mixed reality visual displays. IEICE TRANSACTIONS on Information and Systems, 77(12), pp. 1321-1329, 1994.

[2] P. M. Salkovskis, Psychological treatment of obsessive-compulsive disorder. Psychiatry, vol. 6, no. 6, pp. 229-233, 2007. https://doi.org/10.1016/j.mppsy.2007.03.008

[3] D. H. Barlow, Clinical handbook of psychological disorders: A step-by-step treatment manual. Guilford publications, 2014.

[4] B. Patrão, P. Menezes, and P. Castilho, Development of Mixed Reality Systems to Support Therapies, in Technological Innovation for Cyber-Physical Systems, M. L. CamarinhaMatos, A. J. Falcão, N. Vafaei, and S. Najdi, Eds. Cham: Springer International Publishing, pp. 127-134, 2016. https://doi.org/10.1007/978-3-319-31165-4_13

[5] B. Patrão, S. Pedro, and P. Menezes, Human emotions and physiological signals: A classroom experiment. International Journal of Online and Biomedical Engineering (iJOE), 12(4), pp. 37-39, 2016. https://doi.org/10.3991/ijoe.v12i04.5098

[6] B. Laugwitz, M. Schrepp, and T. Held, Construction and evaluation of a user experience questionnaire. In: Holzinger, A. (Ed.): USAB 2008, LNCS 5298, pp. 63-76, 2008. https://doi.org/10.1007/978-3-540-89350-9 6

[7] J. Lessiter, J. Freeman, E. Keogh, and J. D. Davidoff, A Cross-Media Presence Questionnaire: The ITC Sense of Presence Inventory. Presence: Teleoperators and Virtual Environments, 10(3), pp 282-297, 2001. https://doi.org/10.1162/105474601300343612

\section{$7 \quad$ Authors}

Bruno Patrão is a $\mathrm{PhD}$ student at Electrical and Computer Engineering Department - University of Coimbra, Portugal. He is also member of the Institute of Systems and Robotics - University of Coimbra, Portugal. Email: bpatrao@isr.uc.pt

Paulo Menezes is a Professor at Electrical and Computer Engineering Department - University of Coimbra, Portugal. He is also member of the Institute of Systems and Robotics - University of Coimbra, Portugal. Email: pm@deec.uc.pt

Nuno Gonçalves is a Professor at Electrical and Computer Engineering Department - University of Coimbra, Portugal. He is also member of the Institute of Systems and Robotics - University of Coimbra, Portugal. Email: nunogon@deec.uc.pt

Article submitted 2019-10-15. Resubmitted 2019-12-16. Final acceptance 2019-12-17. Final version published as submitted by the authors. 